\title{
Subharmonicity in von Neumann algebras
}

by

\section{Thomas Ransford and Michel Valley (Québec)}

\begin{abstract}
Let $\mathcal{M}$ be a von Neumann algebra with unit $1_{\mathcal{M}}$. Let $\tau$ be a faithful, normal, semifinite trace on $\mathcal{M}$. Given $x \in \mathcal{M}$, denote by $\mu_{t}(x)_{t \geq 0}$ the generalized $s$ numbers of $x$, defined by

$$
\mu_{t}(x)=\inf \left\{\|x e\|: e \text { is a projection in } \mathcal{M} \text { with } \tau\left(1_{\mathcal{M}}-e\right) \leq t\right\} \quad(t \geq 0) .
$$

We prove that, if $D$ is a complex domain and $f: D \rightarrow \mathcal{M}$ is a holomorphic function, then, for each $t \geq 0, \lambda \mapsto \int_{0}^{t} \log \mu_{s}(f(\lambda)) d s$ is a subharmonic function on $D$. This generalizes earlier subharmonicity results of White and Aupetit on the singular values of matrices.
\end{abstract}

1. Introduction. Let $D$ be a domain in $\mathbb{C}$ and let $M_{n}(\mathbb{C})$ denote the algebra of complex $n \times n$ matrices. It is elementary to show that, if $f: D \rightarrow M_{n}(\mathbb{C})$ is a holomorphic function, then $\lambda \mapsto \log \varrho(f(\lambda))$ is a subharmonic function on $D$, where $\varrho(x)$ denotes the spectral radius of $x$. Vesentini [12] extended this result to holomorphic functions $f: D \rightarrow A$, where $A$ is a general Banach algebra. Vesentini's theorem has many interesting applications; an account of these can be found in Chapter 5 of [1].

More recently, White [13] and Aupetit [2] independently discovered a subharmonicity theorem for the singular values of matrices. Given $x \in$ $M_{n}(\mathbb{C})$, let us write $s_{1}(x), \ldots, s_{n}(x)$ for the singular values of $x$, namely the eigenvalues of $|x|:=\left(x^{*} x\right)^{1 / 2}$ listed in decreasing order. White and Aupetit showed that, if $f: D \rightarrow M_{n}(\mathbb{C})$ is holomorphic, then

$$
\lambda \mapsto \sum_{j=1}^{k} \log s_{j}(f(\lambda))
$$

is subharmonic on $D$, for each $k$ with $1 \leq k \leq n$. Notice that, as pointed out in [2], the individual functions $\lambda \mapsto \log s_{j}(f(\lambda))$ need not be subhar-

2000 Mathematics Subject Classification: Primary 46L10; Secondary 15A18, 31A05.

Key words and phrases: von Neumann algebra, singular value, trace, determinant, subharmonic function.

First author partially supported by grants from NSERC, FQRNT and the Canada research chairs program.

Second author partially supported by an NSERC undergraduate student research award. 
monic if $j \geq 2$. Aupetit proved his theorem in response to a question of O. Nevanlinna, who subsequently exploited the result in his development of a value-distribution theory for matrices (see for example [11, §5]).

The proofs of White and Aupetit (which are essentially the same) rely heavily on the fact that a matrix has a discrete spectrum, and so do not readily generalize to other algebras. There is an alternative method of proof which avoids this problem. The idea is to reduce to the case $k=n$ by considering functions of the form $\lambda \mapsto e f(\lambda) e$, for suitable projections $e$, and then to observe that $\sum_{j=1}^{n} \log s_{j}((f(\lambda))=\log |\operatorname{det}(f(\lambda))|$, which is clearly subharmonic. Our aim in this article is to exploit this general idea to extend the White-Aupetit theorem to the case of holomorphic functions $f: D \rightarrow \mathcal{M}$, where $\mathcal{M}$ is an arbitrary semifinite von Neumann algebra.

2. Notation and statement of results. Let $\mathcal{M}$ be a von Neumann algebra. Denote by $1_{\mathcal{M}}$ the unit of $\mathcal{M}$, and by $\mathcal{M}^{+}$the cone of positive elements of $\mathcal{M}$. A trace on $\mathcal{M}$ is a function $\tau: \mathcal{M}^{+} \rightarrow[0, \infty]$ with the following properties:

- $\tau(x+y)=\tau(x)+\tau(y) \quad\left(x, y \in \mathcal{M}^{+}\right)$

- $\tau(\alpha x)=\alpha \tau(x) \quad\left(x \in \mathcal{M}^{+}, \alpha \in \mathbb{R}^{+}\right)$

- $\tau\left(x^{*} x\right)=\tau\left(x x^{*}\right) \quad(x \in \mathcal{M})$.

A trace $\tau$ is said to be normal if, for every bounded, increasing net $\left\{x_{\alpha}\right\}$, we have $\sup _{\alpha} \tau\left(x_{\alpha}\right)=\tau\left(\sup _{\alpha} x_{\alpha}\right)$. It is finite if $\tau\left(1_{\mathcal{M}}\right)<\infty$, and semifinite if $\tau(x)=\sup \{\tau(y): 0 \leq y \leq x, \tau(y)<\infty\}$. Finally, it is faithful if $\tau(x)=0$ implies $x=0$. A classic reference for von Neumann algebras and traces is [4].

Let $\mathcal{M}$ be a von Neumann algebra, equipped with a faithful, normal, semifinite trace $\tau$. Given $x \in \mathcal{M}$ and $t \in \mathbb{R}^{+}$, we define

$$
\mu_{t}(x):=\inf \left\{\|x e\|: e \text { is a projection in } \mathcal{M} \text { with } \tau\left(1_{\mathcal{M}}-e\right) \leq t\right\} .
$$

This notion was introduced by Murray and von Neumann [10], and has since been further developed by Fack [5], Fack-Kosaki [6] and Hiai-Nakamura [9]. The $\mu_{t}$ are called generalized s-numbers. They generalize the usual notion of singular values in the sense that, if $\mathcal{M}$ is the algebra of bounded operators on a Hilbert space $\mathcal{H}$ and $\tau$ is the usual trace, then, for a compact operator $x \in \mathcal{M}$, we have $\mu_{t}(x)=s_{j}(x)$ for $t \in[j-1, j$ ) (where we agree to define $s_{j}(x)=0$ if $j>\operatorname{dim} \mathcal{H}$ ) (see [5, Exemple 1.2.2]). Many results about singular values extend naturally to the context of generalized $s$-numbers, most notably the Weyl inequalities.

The following result is our main theorem.

THEOREM 2.1. Let $\mathcal{M}$ be a von Neumann algebra, equipped with a faithful, normal, semifinite trace $\tau$. Let $D$ be a complex domain and let 
$f: D \rightarrow \mathcal{M}$ be a holomorphic function. Then, for each $t \geq 0$,

$$
\lambda \mapsto \int_{0}^{t} \log \mu_{s}(f(\lambda)) d s
$$

is a subharmonic function on $D$.

In view of the remarks preceding the theorem, we immediately obtain the following corollary.

Corollary 2.2. Let $\mathcal{H}$ be a Hilbert space, and let $f: D \rightarrow \mathcal{K}(\mathcal{H})$ be a holomorphic function, where $\mathcal{K}(\mathcal{H})$ denotes the ideal of compact operators on $\mathcal{H}$. Then, for each positive integer $k$,

is subharmonic on $D$.

$$
\lambda \mapsto \sum_{j=1}^{k} \log s_{j}(f(\lambda))
$$

In particular, when $\operatorname{dim} \mathcal{H}=n$, we recover the theorem of White and Aupetit mentioned in the introduction (in fact White proved Corollary 2.2 in its general form).

A further example is furnished by the commutative von Neumann algebra $\mathcal{M}=L^{\infty}(\Omega, \nu)$, where $\nu$ is a $\sigma$-finite measure on a set $\Omega$, and $\tau(\phi)=$ $\int \phi d \nu\left(\phi \in \mathcal{M}^{+}\right)$. In this case, $\mu_{s}(\phi)=\phi^{*}(s)$, where $\phi^{*}$ denotes the nondecreasing rearrangement of $|\phi|$ (see [5, Exemple 1.2.1]). We thus obtain the following corollary.

Corollary 2.3. Let $\nu$ be a $\sigma$-finite measure on $\Omega$, and let $\lambda \mapsto \phi_{\lambda}$ : $D \rightarrow L^{\infty}(\Omega, \nu)$ be a holomorphic map. Then, for each $t \geq 0$,

$$
\lambda \mapsto \int_{0}^{t} \log \phi_{\lambda}^{*}(s) d s
$$

is subharmonic on $D$, where $\phi_{\lambda}^{*}$ denotes the non-decreasing rearrangement of $\left|\phi_{\lambda}\right|$.

Theorem 2.1 easily implies the following stronger form of itself.

Corollary 2.4. Let $\mathcal{M}, \tau, D, f$ be as in Theorem 2.1, and let $g$ : $(-\infty, \infty) \rightarrow \mathbb{R}$ be an increasing, convex function. Then, for each $t \geq 0$,

$$
\lambda \mapsto \int_{0}^{t} g\left(\log \mu_{s}(f(\lambda))\right) d s
$$

is subharmonic on $D$.

Proof. Let $\lambda_{0} \in D$, and fix $r$ with $0<r<\operatorname{dist}\left(\lambda_{0}, \partial D\right)$. For $s \geq 0$, define

$$
\phi(s)=\log \mu_{s}\left(f\left(\lambda_{0}\right)\right) \quad \text { and } \quad \psi(s)=\frac{1}{2 \pi} \int_{0}^{2 \pi} \log \mu_{s}\left(f\left(\lambda_{0}+r e^{i \theta}\right)\right) d \theta .
$$


By Theorem 2.1, $\int_{0}^{t} \phi(s) d s \leq \int_{0}^{t} \psi(s) d s$ for all $t \geq 0$. Using [5, Lemme 4.1], we deduce that $\int_{0}^{t} g(\phi(s)) d s \leq \int_{0}^{t} g(\psi(s)) d s$ for all $t \geq 0$. This gives the result.

This strengthened form of the result has an application to finite von Neumann algebras. Suppose that $\tau\left(1_{\mathcal{M}}\right)=1$. Then, by [5, Proposition 1.11],

$$
\int_{0}^{1} \mu_{s}(x) d s=\tau(|x|) \quad(x \in \mathcal{M})
$$

and by [5, Exemple 2.2.2],

$$
\int_{0}^{1} \log \mu_{s}(x) d s=\log \Delta(x) \quad(x \in \mathcal{M}),
$$

where $\Delta$ denotes (the analytic extension of) the Fuglede-Kadison determinant on $\mathcal{M}$ (see [7]).

Corollary 2.5. Let $\mathcal{M}, \tau, D, f$ be as in Theorem 2.1, and suppose in addition that $\tau\left(1_{\mathcal{M}}\right)=1$. Then

$$
\lambda \mapsto \log \tau(|f(\lambda)|) \quad \text { and } \quad \lambda \mapsto \log \Delta(f(\lambda))
$$

are both subharmonic functions on $D$.

Proof. The subharmonicity of $\log \Delta(f(\lambda))$ is an immediate consequence of (3) and Theorem 2.1. Similarly, from (2) and Corollary 2.4 (applied with $\left.g(u)=e^{u}\right)$, it follows that $\tau(|f(\lambda)|)$ is subharmonic on $D$. Repeating the above with $f(\lambda)$ replaced by $e^{p(\lambda)} f(\lambda)$, where $p$ is an arbitrary complex polynomial, we deduce that $\left|e^{p(\lambda)}\right| \tau(|f(\lambda)|)$ is subharmonic on $D$. This is enough to ensure that $\log \tau(|f(\lambda)|)$ is subharmonic on $D$ (see e.g. [1, Theorem A.1.5]).

In fact, the subharmonicity of $\log \Delta(f(\lambda))$ was already known. It was established by Brown as part of a more general result [3, Theorem 3.3], from which he introduced the so-called spectral distribution measure. The paper of Haagerup and Larsen [8] is a recent source of information on Brown's spectral measure.

3. Proof of the main theorem. We now turn to the proof of Theorem 2.1. Throughout this section, we assume that $\mathcal{M}$ is a von Neumann algebra and that $\tau$ is a faithful, normal, semifinite trace on $\mathcal{M}$.

We begin by listing some basic properties of the generalized $s$-numbers $\mu_{s}$.

Proposition 3.1. Let $x, y \in \mathcal{M}$ and $s \in \mathbb{R}^{+}$. Then

(a) $\mu_{s}(x)=\mu_{s}\left(x^{*}\right)=\mu_{s}(|x|)$,

(b) $\mu_{s}(y x) \leq\|y\| \mu_{s}(x)$, 
(c) $\left|\mu_{s}(x)-\mu_{s}(y)\right| \leq\|x-y\|$,

(d) $\mu_{s}\left(x+k 1_{\mathcal{M}}\right) \leq \mu_{s}(x)+k$ for $k \in \mathbb{R}^{+}$, with equality when $x \in \mathcal{M}^{+}$ and $s<\tau\left(1_{\mathcal{M}}\right)$.

Proof. Parts (a)-(c) are in [6, Lemma 2.5]. Part (d) is an easy consequence of [5, Propositions 1.6(iii) and 1.3].

The next result takes care of upper semicontinuity.

Lemma 3.2. Let $f: D \rightarrow \mathcal{M}$ be a continuous function. Then, for each $t \geq 0$, the function $\lambda \mapsto \int_{0}^{t} \log \mu_{s}(f(\lambda)) d s$ is upper semicontinuous on $D$.

Proof. Proposition 3.1(c) shows that the function $\lambda \mapsto \mu_{s}(f(\lambda))$ is continuous. Hence, if $\lambda_{n} \rightarrow \lambda_{0}$ in $D$, then, by Fatou's lemma,

$$
\limsup _{n \rightarrow \infty} \int_{0}^{t} \log \mu_{s}\left(f\left(\lambda_{n}\right)\right) d s \leq \int_{0}^{t} \log \mu_{s}\left(f\left(\lambda_{0}\right)\right) d s .
$$

This proves upper semicontinuity.

For the time being, we make the additional assumption that the trace $\tau$ is finite, i.e. that $\tau\left(1_{\mathcal{M}}\right)<\infty$. In this case, $\tau$ extends to a positive linear functional on the whole of $\mathcal{M}$.

Lemma 3.3. Let $\tau$ be a faithful, normal, finite trace on $\mathcal{M}$. Then

$$
\int_{0}^{\tau\left(1_{\mathcal{M}}\right)} \log \mu_{s}(\exp x) d s=\operatorname{Re} \tau(x) \quad(x \in \mathcal{M}) .
$$

Proof. Notice first that the validity of (4) remains unchanged if we work with the trace $c \tau(c>0)$ instead of $\tau$. Thus, we may as well suppose at the outset that $\tau\left(1_{\mathcal{M}}\right)=1$. The left-hand side of $(4)$ is then equal to $\log \Delta(\exp x)$, where $\Delta$ is the Fuglede-Kadison determinant (see (3)). From [7, Theorem $1(2)]$, we have $\log \Delta(\exp x)=\operatorname{Re} \tau(x)$ for all $x \in \mathcal{M}$. The result follows.

Lemma 3.4. Let $\tau$ be a faithful, normal, finite trace on $\mathcal{M}$, and let $f: D \rightarrow \mathcal{M}$ be a holomorphic function. Then

$$
\lambda \mapsto \int_{0}^{\tau\left(1_{\mathcal{M}}\right)} \log \mu_{s}(f(\lambda)) d s
$$

is a subharmonic function on D.

Proof. For $\varepsilon>0$, define

$$
v_{\varepsilon}(\lambda):=\int_{0}^{\tau\left(1_{\mathcal{M}}\right)} \log \left(\mu_{s}(f(\lambda))+\varepsilon\right) d s \quad(\lambda \in D) .
$$


We prove that each $v_{\varepsilon}$ is a subharmonic function on $D$. The result then follows upon letting $\varepsilon \rightarrow 0$.

Fix $\varepsilon>0$. The upper semicontinuity of $v_{\varepsilon}$ is proved in the same way as in Lemma 3.2. It remains to check the mean-value inequality. Let $\lambda_{0} \in D$. Fix a partial isometry $u_{0} \in \mathcal{M}$ such that $u_{0} f\left(\lambda_{0}\right)=\left|f\left(\lambda_{0}\right)\right|$, and define

$$
w(\lambda):=\int_{0}^{\tau\left(1_{\mathcal{M}}\right)} \log \mu_{s}\left(u_{0} f(\lambda)+\varepsilon 1_{\mathcal{M}}\right) d s \quad(\lambda \in D) .
$$

Using parts (b) and (d) of Proposition 3.1, we see that $w \leq v_{\varepsilon}$ on $D$, with equality at $\lambda_{0}$. We claim that $w$ is actually harmonic in a neighbourhood of $\lambda_{0}$. If so, then, for all small enough $r>0$, we have

$$
v_{\varepsilon}\left(\lambda_{0}\right)=w\left(\lambda_{0}\right)=\frac{1}{2 \pi} \int_{0}^{2 \pi} w\left(\lambda_{0}+r e^{i \theta}\right) d \theta \leq \frac{1}{2 \pi} \int_{0}^{2 \pi} v_{\varepsilon}\left(\lambda_{0}+r e^{i \theta}\right) d \theta,
$$

thereby proving the mean-value inequality.

It remains to justify the claim about $w$. Notice that the spectrum of $u_{0} f\left(\lambda_{0}\right)+\varepsilon 1_{\mathcal{M}}$ is a subset of $[\varepsilon, \infty)$. By the upper semicontinuity of the spectrum, there exists an open disk $D_{0}$ around $\lambda_{0}$ such that the spectrum of $u_{0} f(\lambda)+\varepsilon 1_{\mathcal{M}}$ lies in the open right half-plane for every $\lambda \in D_{0}$. Using the holomorphic functional calculus, we can define $h(\lambda):=$ $\log \left(u_{0} f(\lambda)+\varepsilon 1_{\mathcal{M}}\right)\left(\lambda \in D_{0}\right)$. It follows that

$$
w(\lambda)=\int_{0}^{\tau\left(1_{\mathcal{M}}\right)} \log \mu_{s}(\exp (h(\lambda))) d s=\operatorname{Re} \tau(h(\lambda)) \quad\left(\lambda \in D_{0}\right),
$$

where the second equality is from Lemma 3.3. Since $h$ is holomorphic on $D_{0}$, we deduce that $w$ is harmonic on $D_{0}$, justifying the claim.

The last ingredient is a technique from [5] which permits us to reduce the general case to the case of finite trace. Given a projection $e \in \mathcal{M}$ with $\tau(e)<\infty$, the restriction of $\tau$ to the von Neumann algebra $e \mathcal{M} e$ gives a normal, faithful, finite trace (since the unit of $e \mathcal{M} e$ is $e$ ). We will use the symbol $\mu_{s}^{e}$ to denote the generalized $s$-numbers in $e \mathcal{M} e$.

Lemma 3.5. Let e be a projection in $\mathcal{M}$ with $\tau(e)<\infty$. Then

$$
\mu_{s}^{e}(e x e) \leq \mu_{s}(x) \quad\left(x \in \mathcal{M}, s \in \mathbb{R}^{+}\right) .
$$

Proof. For $x \in \mathcal{M}^{+}$, this is proved in [5, Proposition 1.5(i)]. The general case follows upon observing that, for general $x \in \mathcal{M}$,

$$
\mu_{s}^{e}(e x e)=\mu_{s}^{e}(|e x e|) \leq \mu_{s}^{e}(e|x| e) \leq \mu_{s}(|x|)=\mu_{s}(x) .
$$

Lemma 3.6. Assume that $\mathcal{M}$ has no minimal projections. Let $x \in \mathcal{M}^{+}$ and $t>0$. Suppose that $\mu_{s}(x)>0$ for $0 \leq s<t$. Then there exists 
a projection $e \in \mathcal{M}$, commuting with $x$, such that $\tau(e)=t$ and $\mu_{s}^{e}($ exe $)$ $=\mu_{s}(x)$ for $0 \leq s<t$.

Proof. This is proved in [5, Lemme 1.13], under the additional assumption that $\lim _{s \rightarrow \infty} \mu_{s}(x)=0$. But in fact it is true without this restriction. Indeed, just as in the proof of [6, Lemma 4.1], one can reduce to the case $\mathcal{M}=L^{\infty}(\Omega, \nu)$, where $\nu$ is a non-atomic measure, and $\tau(\phi)=\int \phi d \nu$ $(\phi \in \mathcal{M})$ (see also [6, Remarks 2.3]). In this case $\mu_{s}(\phi)=\phi^{*}(s)$, where $\phi^{*}$ denotes the non-decreasing rearrangement of $|\phi|$.

Given $x, t$ as in the lemma, set $E=\left\{\omega \in \Omega:|x(\omega)|>x^{*}(t)\right\}$ and $F=\left\{\omega \in \Omega:|x(\omega)| \geq x^{*}(t)\right\}$. Then $\nu(E) \leq t \leq \nu(F)$. As $\nu$ is non-atomic, there exists a measurable set $G$ with $E \subset G \subset F$ and $\nu(G)=t$. Let $e$ be the characteristic function of $G$; this satisfies all the conclusions of the lemma.

As remarked in both [5] and [6], the assumption that $\mathcal{M}$ have no minimal projections is not a very serious restriction, because we can always embed $\mathcal{M}$ into $\mathcal{M} \otimes L^{\infty}[0,1]$, extending $\tau$ by taking its tensor product with the trace $\phi \mapsto \int_{0}^{1} \phi$ on $L^{\infty}[0,1]$. The $s$-numbers $\widetilde{\mu}_{s}$ in this new algebra satisfy $\widetilde{\mu}_{s}(x \otimes 1)=\mu_{s}(x)(x \in \mathcal{M})$.

Completion of the proof of Theorem 2.1. Upper semicontinuity has been proved in Lemma 3.2, so it remains to check the mean-value inequality. Let $\lambda_{0} \in D$ and $0<r<\operatorname{dist}\left(\lambda_{0}, \partial D\right)$. We shall show that

$$
\int_{0}^{t} \log \mu_{s}\left(f\left(\lambda_{0}\right)\right) d s \leq \frac{1}{2 \pi} \int_{0}^{2 \pi} \int_{0}^{t} \log \mu_{s}\left(f\left(\lambda_{0}+r e^{i \theta}\right)\right) d s d \theta .
$$

If $\mu_{s}\left(f\left(\lambda_{0}\right)\right)=0$ for some $s<t$, then the left-hand side is $-\infty$, and the inequality is trivially satisfied. So we can suppose that $\mu_{s}\left(f\left(\lambda_{0}\right)\right)>0$ for $0 \leq s<t$. By the remark above, we may also assume that the algebra $\mathcal{M}$ has no minimal projections. Hence, by Lemma 3.6, there exists a projection $e \in \mathcal{M}$, commuting with $\left|f\left(\lambda_{0}\right)\right|$, such that $\tau(e)=t$ and $\mu_{s}^{e}\left(e f\left(\lambda_{0}\right) e\right)=$ $\mu_{s}\left(f\left(\lambda_{0}\right)\right)$ for $0 \leq s<t$. Therefore

$$
\begin{aligned}
\int_{0}^{t} \log \mu_{s}\left(f\left(\lambda_{0}\right)\right) d s & =\int_{0}^{t} \log \mu_{s}^{e}\left(e f\left(\lambda_{0}\right) e\right) d s \\
& \leq \frac{1}{2 \pi} \int_{0}^{2 \pi} \int_{0}^{t} \log \mu_{s}^{e}\left(e f\left(\lambda_{0}+r e^{i \theta}\right) e\right) d s d \theta \\
& \leq \frac{1}{2 \pi} \int_{0}^{2 \pi} \int_{0}^{t} \log \mu_{s}\left(f\left(\lambda_{0}+r e^{i \theta}\right)\right) d s d \theta
\end{aligned}
$$

where the first inequality is by Lemma 3.4 and the second by Lemma 3.5. This completes the proof. 


\section{References}

[1] B. Aupetit, A Primer on Spectral Theory, Universitext, Springer, New York, 1991.

[2] -, On log-subharmonicity of singular values of matrices, Studia Math. 122 (1997), 195-200.

[3] L. G. Brown, Lidskii's theorem in the type II case, in: Geometric Methods in Operator Algebras, H. Araki and E. Effros (eds.), Pitman Res. Notes Math. Ser. 123, Longman, New York, 1986, 1-35.

[4] J. Dixmier, Les algèbres d'opérateurs dans l'espace hilbertien (algèbres de von Neumann), Gauthier-Villars, Paris, 1969.

[5] T. Fack, Sur la notion de valeur caractéristique, J. Operator Theory 7 (1982), 307-333.

[6] T. Fack and H. Kosaki, Generalized s-numbers of $\tau$-measurable operators, Pacific J. Math. 123 (1986), 269-300.

[7] B. Fuglede and R. Kadison, Determinant theory in finite factors, Ann. of Math. 55 (1952), 520-530.

[8] U. Haagerup and F. Larsen, Brown's spectral distribution measure for R-diagonal elements in finite von Neumann algebras, J. Funct. Anal. 176 (2000), 331-367.

[9] F. Hiai and Y. Nakamura, Majorizations for generalized s-numbers in semifinite von Neumann algebras, Math. Z. 195 (1987), 17-27.

[10] F. J. Murray and J. von Neumann, On rings of operators, Ann. of Math. 37 (1936), $116-229$.

[11] O. Nevanlinna, Meromorphic Functions and Linear Algebra, Fields Inst. Monogr., Amer. Math. Soc., Providence, RI, 2003.

[12] E. Vesentini, On the subharmonicity of the spectral radius, Boll. Un. Mat. Ital. 4 (1968), 427-429.

[13] M. C. White, Analytic multivalued functions and symmetrically normed ideals, Ph.D. thesis, Univ. of Cambridge, 1989.

Département de mathématiques et de statistique

Université Laval

Québec (QC), Canada G1K 7P4

E-mail: ransford@mat.ulaval.ca

mvalley@mat.ulaval.ca

Received November 28, 2003

Revised version April 25, 2005 